\title{
BMJ Open Healthcare resource utilisation for chronic kidney disease and other major non-communicable chronic diseases in China: a cross-sectional study
}

\author{
Chao Yang (D) , ${ }^{1,2,3}$ Jianyan Long, ${ }^{4}$ Ying Shi, ${ }^{5}$ Zhiye Zhou, ${ }^{5}$ Jinwei Wang (D) ,, 2 \\ Ming-Hui Zhao, ${ }^{1,2,6}$ Haibo Wang, ${ }^{4,7}$ Luxia Zhang, ${ }^{1,3,7}$ Josef Coresh $^{8}$
}

To cite: Yang C, Long J, Shi Y, et al. Healthcare resource utilisation for chronic kidney disease and other major non-communicable chronic diseases in China: a crosssectional study. BMJ Open 2022;12:e051888. doi:10.1136/ bmjopen-2021-051888

\section{- Prepublication history and} additional supplemental material for this paper are available online. To view these files, please visit the journal online (http://dx.doi.org/10.1136/ bmjopen-2021-051888).

Received 31 March 2021 Accepted 14 December 2021

Check for updates

(C) Author(s) (or their employer(s)) 2022. Re-use permitted under CC BY-NC. No commercial re-use. See rights and permissions. Published by BMJ.

For numbered affiliations see end of article.

Correspondence to Dr Luxia Zhang; zhanglx@bjmu.edu.cn and Dr Haibo Wang;

haibo@mail.harvard.edu

\section{ABSTRACT}

Objective To evaluate the healthcare resource utilisation for chronic kidney disease (CKD) and other major noncommunicable chronic diseases (NCDs) in China.

Design A cross-sectional study.

Setting A national inpatient database of tertiary hospitals in China.

Participants The study included a total of 19.5 million hospitalisations of adult patients from July 2013 to June 2014. Information on CKD and other major NCDs, including coronary heart disease (CHD), stroke, hypertension, diabetes, chronic obstructive pulmonary disease (COPD) and cancer, was extracted from the unified discharge summary form.

Outcome measures Cost, length of hospital stay and in-hospital mortality.

Results The percentages of hospitalisations with CKD, CHD, stroke, hypertension, diabetes, COPD and cancer were $4.5 \%, 9.2 \%, 8.2 \%, 18.8 \%, 7.9 \%, 2.3 \%$ and $19.4 \%$, respectively. For each major NCD, the presence of CKD was independently associated with longer hospital stay, with increased percentages ranging from $7.69 \%(95 \% \mathrm{Cl}$ $7.11 \%$ to $8.28 \%$ ) for stroke to $21.60 \%$ ( $95 \% \mathrm{Cl} 21.09 \%$ to $22.10 \%)$ for CHD. Hospital mortality for other NCDs was also higher in the presence of CKD, with fully adjusted relative risk ranging from $1.91(95 \% \mathrm{Cl} 1.82$ to 1.99$)$ for stroke to 2.65 (95\% Cl 2.55 to 2.75 ) for cancer. Compared with other NCDs, CKD was associated with the longest hospital stay (22.1\% increase) and resulted in the second highest in-hospital mortality, only lower than that of cancer (relative risk, 2.23 vs 2.87, respectively).

Conclusions The presence of diagnosed CKD alongside each major NCD was associated with an additional burden on the healthcare system. Healthcare resource utilisation and prognosis of CKD were comparable with those of other major NCDs, which highlights the importance of CKD as a major public health burden.

\section{BACKGROUND}

There is a rising burden of non-communicable diseases (NCDs) at a global level. Two of three deaths each year are attributable to NCDs, and four-fifths of NCD-related deaths occur in low-income and middle-income countries. ${ }^{1}$ This exerts a significant influence
Strengths and limitations of this study

- This is the first national study placing the chronic kidney disease burden on hospital stay and mortality in the full context of all major non-communicable chronic diseases (NCDs).

- The study has a large sample size and wide geographical coverage.

- Only tertiary hospitals were included, which might have led to population selection bias.

- Diagnosis of major NCDs was based on the International Classification of Diseases-10 coding, with low sensitivity.

- Data on estimated glomerular filtration rate or proteinuria and information on medications were not available.

on the healthcare system and costs worldwide. $^{2}$ Taking action against NCDs is, therefore, an economic imperative. ${ }^{3}$ The priority actions for the NCD crisis were released by the United Nations High-Level Meeting in 2011 , focusing on the prevention and control of heart disease, stroke, cancer, diabetes and chronic respiratory diseases. ${ }^{1}$ World leaders also committed to reduce premature deaths from NCDs by one-third by $2030 .^{3}$

Over the past decade, chronic kidney disease (CKD) has been recognised as a major public health issue worldwide, with an estimated prevalence of more than $10 \% .{ }^{45} \mathrm{By}$ 2040, CKD is estimated to become the fifth leading cause of early death globally-one of the largest projected increases of any major cause of death. ${ }^{6}$ In China, CKD is prevalent and is associated with more severe comorbidities and higher medical expenditures. ${ }^{78}$ However, unlike other NCDs, such as diabetes, the awareness of CKD is low among patients and healthcare providers. ${ }^{9}$ Besides, CKD is a key determinant of poor health outcomes of major NCDs and a risk multiplier 
in patients with cardiovascular disease (CVD), diabetes and hypertension. ${ }^{10}$ Due to the fact that the kidneys are usually target organs of systemic vascular, haemodynamic, metabolic and inflammatory disorders, the pandemic of NCDs also leads to a persistently increasing morbidity of CKD. ${ }^{11}$ There is accumulating evidence that major NCDs, including heart disease and stroke, have poor prognosis in the presence of CKD. ${ }^{12-14}$ Moreover, CKD has been reported to be associated with reduced implementation of best clinical practice, which could lead to suboptimal treatment and adverse outcomes, especially for patients with CVD. ${ }^{15}$

Although CKD is a global health issue, to the best of our knowledge, there have been neither large-scale studies to quantitatively evaluate the burden of CKD on all major NCDs nor studies comparing the burden of CKD with other NCDs. The evidence for healthcare resource utilisation associated with CKD and other major NCDs at a national level is limited, which impedes the development of effective preventive strategies. In addition, little is known about the status of cardiac procedures for patients with CKD in China. Hence, we initiated this observational study to comprehensively and quantitatively evaluate the healthcare resource utilisation and prognosis of CKD and other NCDs, as well as to compare the burden of kidney diseases on the healthcare system with other NCDs among hospitalised patients in China.

\section{METHODS}

\section{Study population}

The Hospital Quality Monitoring System (HQMS) is a mandatory patient-level national database for hospital accreditation under the authority of the National Health Commission of the People's Republic of China. Details of HQMS have been described elsewhere. ${ }^{16}{ }^{17}$ In brief, all tertiary hospitals in China have been requested to submit electronic inpatient discharge records to the HQMS on a daily basis. Unlike the Western medical system, tertiary hospitals in China provide primary, secondary and tertiary care and have exposure to a nationwide patient population. Patient-level data were collected from uniform front pages of hospitalisation medical records. Altogether 353 variables, including demographic characteristics, clinical diagnoses, procedures, pathology diagnoses and expenditure breakdowns, were collected. All personal information has already been de-identified to protect patient privacy. Diagnoses were coded based on the International Classification of Diseases-10 (ICD-10) coding system by certified professional medical coders at every hospital. As part of stringent standard practice in China, inpatient discharge records have legal validity and must be filed by the caregiving doctors who have the most accurate and comprehensive understanding of the patient's medical condition.

A total of 19518990 records of adult inpatients admitted from 1 July 2013 to 30 June 2014 in 29 provinces (excluding Hong Kong, Macao, Taiwan, Tibet and
Ningxia) were included in this cross-sectional study, with certain patients having more than one hospitalisation. During this period, 715 tertiary hospitals in China, accounting for $44 \%$ of all tertiary hospitals in the country, had submitted inpatient records to the HQMS database. Hospitalisations were used in the present analysis since they are more relevant to the discussion of 'disease burden'. ${ }^{18}$

\section{Definition of CKD}

The ICD-10 coding of discharge diagnoses was used to identify patients with CKD. ${ }^{1617}$ Hospitalisations of patients with at least one of the following diagnoses (in both primary diagnosis and secondary diagnoses) were identified as having CKD (relevant ICD-10 coding in online supplemental appendix 1): glomerulonephritis, diabetic nephropathy, hypertensive nephropathy, obstructive nephropathy, renal cancer, tubulointerstitial nephritis, kidney disease secondary to autoimmune diseases and kidney failure of unknown reason. Among patients' records with CKD, only $14 \%$ had information on CKD staging.

\section{Validation study}

Since the laboratory results are not included in the HQMS data set, we performed a validation study using 67376 hospitalisations in three hospitals in the HQMS. These were the pilot hospitals that accomplished the HQMS phase 2 data collection expansion to the data of laboratory tests and medications. ${ }^{19}$ Estimated glomerular filtration rate (eGFR) was calculated using the Modification of Diet in Renal Disease Study equation tailored to Chinese patients with CKD. ${ }^{20}$ Proteinuria was defined by urinary albumin to creatinine ratio $\geq 30 \mathrm{mg} / \mathrm{g}$ creatinine and/or urinary protein $\geq$ trace in the urine routine test. CKD was defined as the presence of proteinuria and/or eGFR less than $60 \mathrm{~mL} / \mathrm{min} / 1.73 \mathrm{~m}^{2}$. Compared with the gold standard, the sensitivity and specificity of CKD identification by ICD-10 coding were $34.2 \%$ and $97.8 \%$, respectively. ${ }^{19}$

\section{Definition of other NCDs}

Major NCDs, including coronary heart disease (CHD), stroke, hypertension, diabetes, chronic obstructive pulmonary disease (COPD) and cancer, ${ }^{1}$ were identified by the ICD-10 coding of hospital discharge diagnoses (in both primary and secondary diagnoses; relevant ICD-10 coding in online supplemental appendix 1). Information on coronary angiography (CAG), percutaneous coronary intervention (PCI) and coronary artery bypass grafting was obtained from the procedure coding of the inpatient discharge records for patients with CHD. For patients with multiple NCD diagnoses, each relevant diagnosis was identified and counted when stratified by the above NCDs.

\section{Other covariates}

Individual variables including age, sex, occupation (professional, worker, farmer, retired, unemployed and others), type of health insurance (basic medical 
insurance, New Rural Cooperative Medical Care, other types of insurance and uninsured), type of admission (emergency, routine and others) and intensive care unit (ICU) stay were collected from the HQMS database.

\section{Outcomes}

Hospitalisation costs and length of hospital stay, which are both important indicators of healthcare resource utilisation, were acquired from the clinical charts in electronic inpatient discharge records. The total cost of the patient's hospitalisation was jointly borne by the patient, the government and related insurers, and included costs for prescribed drugs, hospital beds, laboratory tests, medical examinations and surgical costs. The total cost information was summarised and reported by each hospital and we extracted it directly from the HQMS database. Information on in-hospital mortality was also collected to assess the prognosis of patients. Survival status of each patient was verified based on discharge status and combined with information on autopsy.

\section{Statistical analysis}

General characteristics stratified by the presence of CKD for each NCD were described. Continuous data were presented as mean $\pm \mathrm{SD}$ or as median (IQR) for highly skewed variables. Categorical variables were presented as proportions. All analyses were based on hospitalisations (patient records), not individuals.

The effects of CKD on healthcare resource utilisation for each NCD were analysed using generalised regression models for log-transformed cost and length of stay with log-link function and gamma distribution. Due to the low mortality and large sample size, Poisson regression model was used to evaluate the burden of CKD and other NCDs on in-hospital mortality. Per cent of change ( $\%$ change) and relative risk (RR) with $95 \%$ CI were calculated as $\exp (\beta)-1$ from the generalised regression model and $\exp (\beta)$ from the Poisson regression model, respectively. For the purpose of assessing additional effects of CKD, covariates included in the model for the sub-data set of each NCD were age (5-year categories, except for 18-25 years and $>80$ years), sex (male vs female), type of health insurance (dummy variable), type of admission (dummy variable), ICU stay (yes vs no), presence of CKD (yes vs no), presence of other NCDs (yes vs no), pneumonia (yes vs no) and sepsis (yes vs no).

Then, the presence of CKD and other NCDs was included in the models simultaneously, using the data of all hospitalised patients, in order to compare the effect of CKD with that of other NCDs on outcome variables. Similar covariates were used as described previously.

For hospitalised patients with CHD, the percentages of those with CAG and PCI were reported among different clinical types (including ST segment elevation myocardial infarction, non-ST segment elevation myocardial infarction and non-acute coronary syndrome), stratified by the presence of CKD. Among 18315 hospitalisations with
CHD and with available CKD staging, the percentages of those with CAG and PCI were also reported.

Sensitivity analysis was conducted among patients with CKD stages $3-5$ to further determine the healthcare resource utilisation for CKD (online supplemental appendices 2 and 3). All analyses were performed using SAS V.9.4 software. Due to the large sample size of our study, $p$ values were not reported for between-group comparisons.

\section{Patient and public involvement statement}

Patients or the public were not involved in this study.

\section{RESULTS}

Among 19.5 million hospitalisations, the percentages of diagnosed CKD, CHD, stroke, hypertension, diabetes, COPD and cancer were 4.5\%, 9.2\%, 8.2\%, 18.8\%, 7.9\%, $2.3 \%$ and $19.4 \%$, respectively (table 1 ). For each major NCD, individuals with CKD were older (except for hypertension), were more often male and urban residents, more often stayed in ICU, and had infectious diseases such as pneumonia and sepsis (except for COPD).

For each major NCD, the presence of CKD was independently associated with longer hospital stay, with increased percentages ranging from $7.69 \%$ (95\% CI $7.11 \%$ to $8.28 \%$ ) for stroke to $21.60 \%$ (95\% CI $21.09 \%$ to $22.10 \%$ ) for CHD (table 2). After adjusting for potential confounders, the presence of CKD was associated with higher costs for stroke (11.15\%; 95\% CI $10.31 \%$ to $11.99 \%)$, COPD (19.46\%; $95 \%$ CI $17.97 \%$ to $20.97 \%$ ) and cancer $(18.88 \%$; $95 \%$ CI $17.98 \%$ to $19.78 \%)$. In contrast, the costs were slightly lower for CHD (acute coronary syndrome or non-acute coronary syndrome) $(-1.08 \%$; $95 \%$ CI $-1.71 \%$ to $-0.45 \%)$, hypertension $(-0.22 \%$; $95 \%$ CI $-0.60 \%$ to $0.16 \%)$ and diabetes $(-0.97 \%$; $95 \%$ CI $-1.43 \%$ to $-0.51 \%$ ) in the presence of CKD (table 2). If patients with CHD were excluded, the presence of CKD was associated with increased costs for patients with hypertension and diabetes, with a fully adjusted change of $2.70 \%$ (95\% CI $2.24 \%$ to $3.15 \%$ ) and $4.11 \%$ (95\% CI $3.56 \%$ to $4.66 \%$ ), respectively. In-hospital mortality was also higher in the presence of CKD for major NCDs, with fully adjusted RR ranging from 1.91 (95\% CI 1.82 to 1.99, for stroke; $95 \%$ CI 1.85 to 1.97 , for hypertension) to 2.65 (95\% CI 2.55 to 2.75 , for cancer) (table 2).

Among hospitalisations with CHD, the percentages of individuals with CAG and PCI were substantially lower for those with CKD (figure 1). A similar pattern was observed for coronary artery bypass grafting, which was $0.3 \%$ and $1.0 \%$ for CHD patients with or without CKD, respectively. Even for those with acute coronary syndrome or cardiogenic shock - a strong indication for emergency $\mathrm{PCI}^{21}$ - the trend was still similar: $11.9 \%$ vs $22.2 \%$ for ST elevated myocardial infarction and $3.5 \%$ vs $7.3 \%$ for non-ST elevated myocardial infarction. Furthermore, among hospitalisations with available CKD staging and with CHD, the percentages of individuals with CAG and 


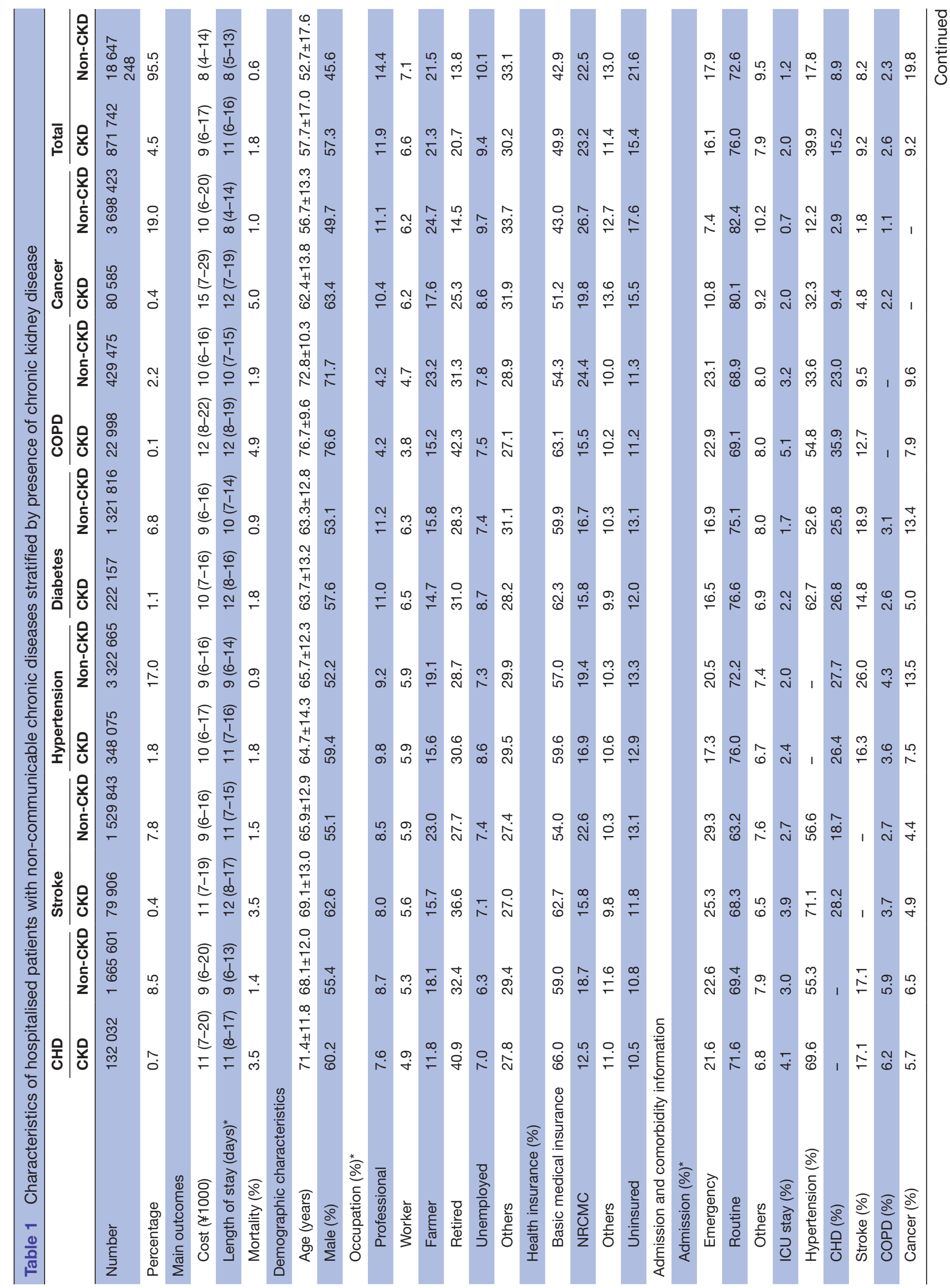




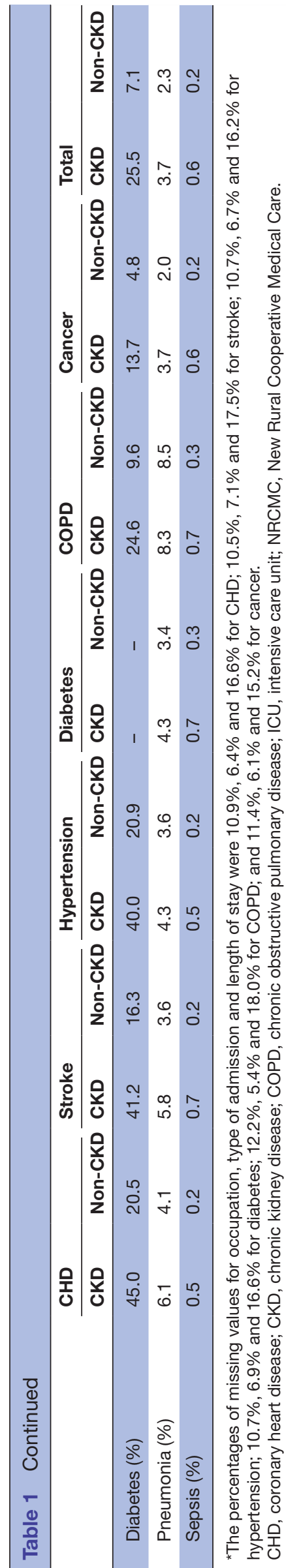

PCI were lower even for stages 1 and 2, compared with those without CKD and with CHD (figure 1).

Compared with other NCDs, CKD had the highest increase in length of hospital stay $(22.09 \%$; $95 \%$ CI $21.87 \%$ to $22.32 \%$ ) (table 3 ). Cancer and CHD were associated with the highest contribution to increased costs for hospitalised patients, which was $39.47 \%$ (95\% CI 39.29\% to $39.65 \%$ ) and $30.95 \%$ (95\% CI $30.71 \%$ to $31.19 \%$ ), respectively. CKD was associated with $6.82 \%(95 \%$ CI $6.56 \%$ to $7.08 \%$ ) increased costs. Furthermore, CKD resulted in the second highest in-hospital mortality (RR, $2.23 ; 95 \%$ CI 2.19 to 2.28 ), which was only lower than that of cancer (RR, 2.87; 95\% CI 2.83 to 2.91 ).

\section{DISCUSSION}

This study is the first national quantitative study on the burden of CKD and other major NCDs on the healthcare system in China. The findings of this study indicated that the presence of CKD was associated with additional burden on the healthcare system and increased in-hospital mortality for each major NCD, despite evidence of underutilisation of cardiac procedures in the presence of CKD. Furthermore, healthcare resource utilisation for CKD was comparable with those of other major NCDs in this large national sample of 19.5 million hospitalisations.

Studies regarding utilisation of healthcare resources for CKD mostly focused on end-stage kidney disease (ESKD). ${ }^{22-24}$ The US Renal Data System 2019 Annual Data Report showed that the costs for both CKD and ESKD were in excess of $\$ 120$ billion in 2017 , and the latter accounted for more than $7.2 \%$ of the total Medicare expenditure. ${ }^{25}$ Our study revealed that CKD was associated with a $6.8 \%$ increase in costs, which was higher than that of hypertension, diabetes and COPD. Moreover, for each major NCD, the presence of CKD led to longer hospital stay, which is also an important marker of healthcare resource utilisation. Furthermore, considering the evidence of therapeutic nihilism for patients with CKD (documented for cardiac revascularisation) and the insensitivity of diagnostic codes for CKD, the burden of CKD on the healthcare system might be underestimated. A recent systematic review has also shown that the risks of adverse cardiovascular outcomes increase with CKD and are associated with substantial additional costs and resource utilisation. ${ }^{26}$

Several previous studies have reported that both the general and high-risk populations have poor prognoses in the presence of kidney diseases. For example, using the data of 1.1 million adults within a large integrated healthcare system, Go et $a l^{12}$ observed increased risks of all-cause mortality, CVD and hospitalisation associated with reduced kidney function. A recent meta-analysis has reported that both reduced eGFR and elevated urinary albumin to creatinine ratio are associated with increased all-cause and cardiovascular mortality in the general population. ${ }^{27}$ Similarly, using data from over 40 cohorts involving one million participants, it has been reported 
Table 2 Effects of chronic kidney disease on length of stay, costs and in-hospital mortality for each non-communicable disease

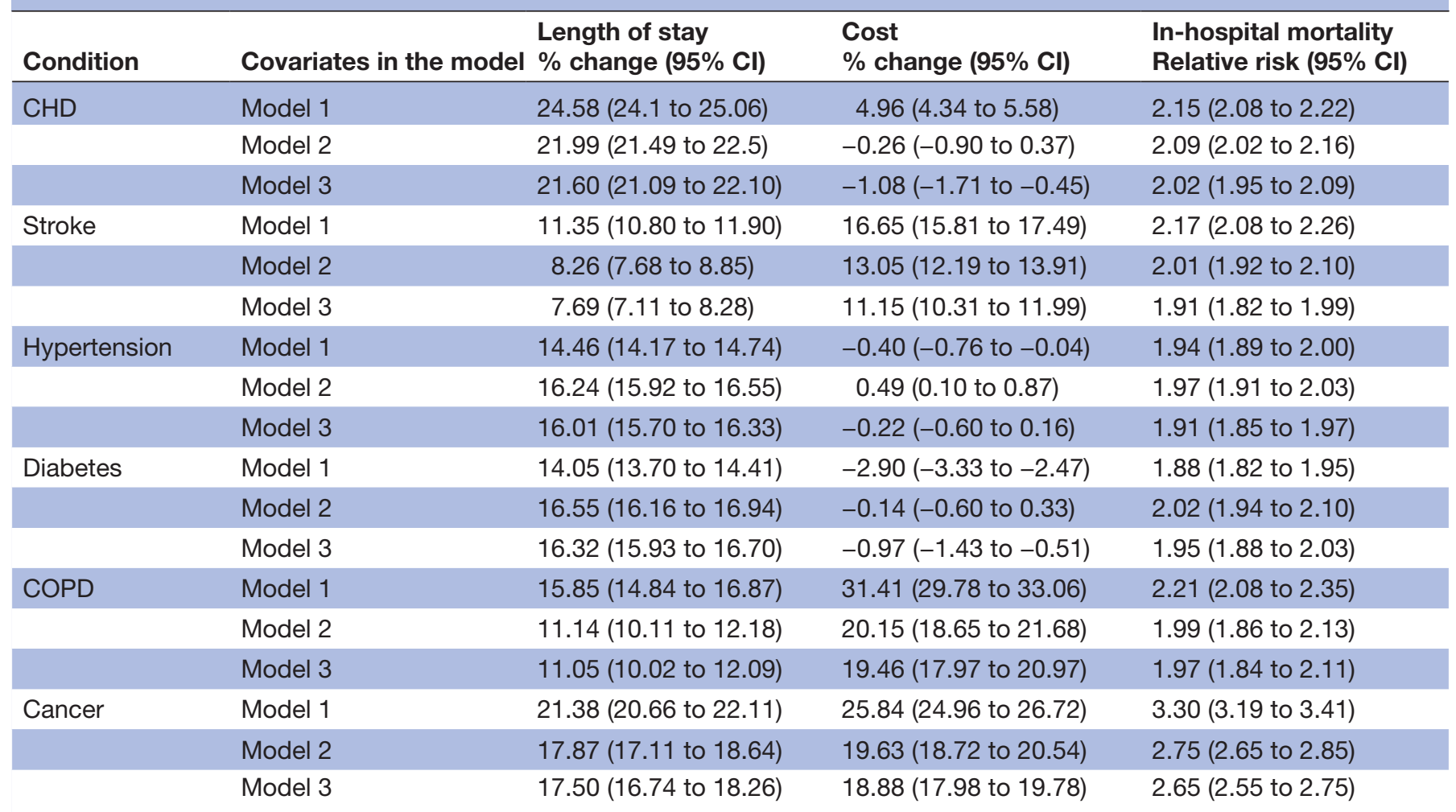

Numbers in the table are per cent changes/relative risk of patient records with CKD compared with patient records without CKD for each non-communicable disease. Per cent of change (\% change) and relative risk with $95 \% \mathrm{Cl}$ were calculated as $\exp (\beta)-1$ from the generalised regression model and $\exp (\beta)$ from the Poisson regression model, respectively.

Covariates in model 1 included age and sex.

Covariates in model 2 included age, sex, occupation, type of health insurance, type of admission, intensive care unit stay, and presence of CHD, stroke, hypertension, diabetes, COPD and cancer (except for the disease used to define the subgroup).

Covariates in model 3 included covariates in model 2, plus presence of sepsis and pneumonia.

$\mathrm{CHD}$, coronary heart disease; CKD, chronic kidney disease; COPD, chronic obstructive pulmonary disease.

that the adjusted HR for all-cause mortality at an eGFR of $45 \mathrm{~mL} / \mathrm{min} / 1.73 \mathrm{~m}^{2}$ was 1.24 (95\% CI 1.11 to 1.39 ) for those with hypertension ${ }^{28}$ and was 1.35 (95\% CI 1.18 to 1.55 ) for those with diabetes, ${ }^{29}$ compared with those with eGFR of $95 \mathrm{~mL} / \mathrm{min} / 1.73 \mathrm{~m}^{2}$. In this study, we further expanded the observation to a range of other major NCDs, including CHD, stroke, COPD and cancer. We found that CKD was consistently associated with increased in-hospital mortality for each major NCD, and its impact was higher compared with that of the majority of NCDs, even comparable with that of cancer. These findings further highlight the importance of CKD as a major health burden and are consistent with the implications of Global Burden of Disease, Injuries, and Risk Factors Study, which has shown the high burden and rapid growth of CKD as a direct cause of morbidity and mortality. ${ }^{4}$

The potential mechanisms for the association of CKD and adverse outcomes are complex and not fully understood. A previous study indicated that impaired kidney function could lead to multiple adverse systemic alterations, including inappropriate activation of the reninangiotensin system, catalytic iron-dependent oxidative stress, endothelial dysfunction and inflammation. ${ }^{14}$ In addition, for patients whose CKD is a result of a systemic disease such as diabetes, kidney diseases might only serve as a marker of severity of the target organ damage.

Another explanation for the adverse outcomes observed in patients with CKD might be that the presence of CKD is associated with reduced implementation of best practice. Although the accumulating evidence and the Kidney Disease: Improving Global Outcomes guidelines with international consensus suggest that the level of care for ischaemic heart disease offered to patients with CKD should not be prejudiced by their CKD ${ }^{30}$ our data on revascularisation indicate that the cardiac treatment for patients with CKD is suboptimal. Underutilisation of aspirin, beta-blockers, ACE inhibitors, glycoprotein IIb/ IIIa receptor antagonists and thrombolytic therapy has been observed in patients with CKD due to concerns of bleeding risk, worsening of kidney function and comorbidities. ${ }^{15}$ A recent study from the USA indicated that, compared with those without CKD, patients with CKD reported more medication use for cardiovascular risk factors but had poorer risk factor control. ${ }^{31}$ In our study, the percentages of hospitalisations with $\mathrm{CAG}$ and PCI were much lower for those with CKD, even for those with 


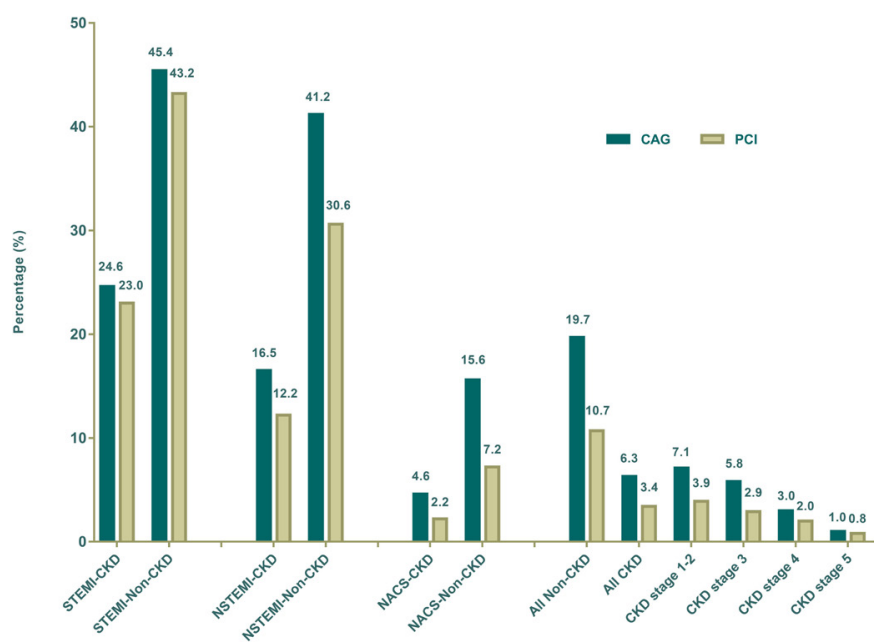

Figure 1 Percentages of hospitalisations with CAG and $\mathrm{PCl}$ for individuals with coronary heart disease. The number of hospitalisations with or without CKD was 3116 and 68616 for STEMI, respectively; 2645 and 25057 for NSTEMI, respectively; and 124388 and 1519864 for NACS, respectively. For 18315 hospitalisations with available CKD staging, $15.8 \%, 24.6 \%, 16.8 \%$ and $42.9 \%$ of individuals were in stages $1-2,3,4$ and 5 , respectively. CAG, coronary angiography; CKD, chronic kidney disease; NACS, nonacute coronary syndrome; NSTEMI, non-ST segment elevation myocardial infarction; $\mathrm{PCl}$, percutaneous coronary intervention; STEMI, ST segment elevation myocardial infarction. relatively well-preserved kidney function, consistent with the practice outside of China. ${ }^{15}$ A recent meta-analysis has revealed that for patients with predialysis CKD and with unstable angina or non-ST segment elevation myocardial infarction, an early invasive strategy is associated with risk of rehospitalisation and reduced risk of death and non-fatal reinfarction. ${ }^{32}$ However, the risk of adverse clinical events after coronary revascularisation and the percentage of later additional PCI are also increased in patients with CKD. ${ }^{33}{ }^{34}$ Hence, a multidisciplinary team involving various subspecialties is needed to improve the quality of care for patients with CKD, and appropriate management by primary care clinicians is necessary to prevent CKD-associated adverse outcomes. ${ }^{35}$

To our knowledge, this is the first study that places the CKD burden on hospital stay and mortality in the full context of all major NCDs in a national database with large sample size and wide geographical coverage, which enabled us to analyse the burden of multiple major NCDs simultaneously. However, our study does have some limitations. First, although our data were restricted to hospitalised patients, hospitalisation inherently reflects the indication for admission and referral as well as disease burden. Only tertiary hospitals were included in our analyses, which might have led to population selection bias; nevertheless, 715 hospitals in our study covered almost all provinces of China and included all types of tertiary

\begin{tabular}{|c|c|c|c|c|}
\hline Condition & $\begin{array}{l}\text { Covariates in the } \\
\text { model }\end{array}$ & $\begin{array}{l}\text { Length of stay } \\
\% \text { change }(95 \% \mathrm{Cl})\end{array}$ & $\begin{array}{l}\text { Cost } \\
\% \text { change }(95 \% \mathrm{Cl})\end{array}$ & $\begin{array}{l}\text { In-hospital mortality } \\
\text { Relative risk }(95 \% \mathrm{Cl})\end{array}$ \\
\hline \multirow[t]{2}{*}{ CKD } & Model 1 & 22.34 (22.14 to 22.55$)$ & 6.49 (6.25 to 6.73$)$ & 2.31 (2.27 to 2.35$)$ \\
\hline & Model 2 & 22.09 (21.87 to 22.32$)$ & $6.82(6.56$ to 7.08$)$ & 2.23 (2.19 to 2.28$)$ \\
\hline \multirow[t]{2}{*}{ CHD } & Model 1 & $-5.19(-5.31$ to -5.07$)$ & 27.72 (27.51 to 27.94$)$ & 1.36 (1.34 to 1.38$)$ \\
\hline & Model 2 & $-7.31(-7.44$ to -7.18$)$ & 30.95 (30.71 to 31.19$)$ & 1.31 (1.29 to 1.33 ) \\
\hline \multirow[t]{2}{*}{ Stroke } & Model 1 & 16.12 (15.97 to 16.27$)$ & 9.29 (9.10 to 9.48$)$ & 1.58 (1.56 to 1.60$)$ \\
\hline & Model 2 & 17.34 (17.17 to 17.51$)$ & 9.90 (9.69 to 10.11$)$ & 1.70 (1.67 to 1.73$)$ \\
\hline \multirow[t]{2}{*}{ Hypertension } & Model 1 & 5.49 (5.39 to 5.59$)$ & 8.90 (8.77 to 9.04$)$ & $0.93(0.92$ to 0.94$)$ \\
\hline & Model 2 & 1.81 (1.69 to 1.92$)$ & 4.85 (4.70 to 5.01$)$ & 0.76 (0.75 to 0.77$)$ \\
\hline \multirow[t]{2}{*}{ Diabetes } & Model 1 & 11.36 (11.22 to 11.51$)$ & 5.05 (4.87 to 5.23$)$ & $1.13(1.11$ to 1.15$)$ \\
\hline & Model 2 & 8.96 (8.80 to 9.12$)$ & 1.00 (0.81 to 1.20$)$ & 0.99 (0.97 to 1.01$)$ \\
\hline \multirow[t]{2}{*}{ COPD } & Model 1 & 3.89 (3.65 to 4.14$)$ & $-9.00(-9.29$ to -8.71$)$ & 1.31 (1.28 to 1.34$)$ \\
\hline & Model 2 & 5.77 (5.50 to 6.04$)$ & $-8.67(-8.98$ to -8.36$)$ & 1.24 (1.21 to 1.27$)$ \\
\hline \multirow[t]{2}{*}{ Cancer } & Model 1 & 8.90 (8.80 to 9.00$)$ & 27.40 (27.25 to 27.55$)$ & 2.00 (1.97 to 2.02$)$ \\
\hline & Model 2 & 13.90 (13.79 to 14.02$)$ & 39.47 (39.29 to 39.65 ) & 2.87 (2.83 to 2.91$)$ \\
\hline
\end{tabular}

Numbers in the table are per cent changes/relative risks of patient records with corresponding disease compared with patient records without corresponding disease. Per cent of change (\% change) and relative risk with $95 \% \mathrm{Cl}$ were calculated as exp( $\beta)$ - 1 from the generalised regression model and $\exp (\beta)$ from the Poisson regression model, respectively.

Covariates in model 1 included age and sex.

Covariates in model 2 included age, sex, occupation, type of health insurance, type of admission, intensive care unit stay, presence of CKD, $\mathrm{CHD}$, stroke, hypertension, diabetes, COPD and cancer, and presence of sepsis and pneumonia.

$\mathrm{CHD}$, coronary heart disease; CKD, chronic kidney disease; COPD, chronic obstructive pulmonary disease. 
hospitals. Second, the diagnosis of CKD and major NCDs in hospitalised patients was based on ICD-10 coding, which has low sensitivity. Severe cases of CKD were likely to be diagnosed, which might affect the extrapolation of the results and lead to a potential overestimation of the excess risk associated with CKD. However, the high specificity of CKD identification (97.8\%) is the strength of our study. Third, the low usage of CKD staging codes was a limitation that should be recognised. Further evaluation of healthcare utilisation in different CKD stages is worthwhile. Fourth, since hospitalisations were identified using both primary and secondary diagnoses, the direct contribution of specific NCDs to health resource utilisation might have been compromised. All analyses were based on hospitalisations, and cases reported in the results were not mutually exclusive, which may also affect the estimations of the true burden of disease. Finally, data on eGFR or proteinuria and information on medications used and severity of major NCDs were not available for all patient records in our data set.

\section{CONCLUSIONS}

In a systematic national assessment of diagnosed NCDs over 19.5 million hospitalisations in China, we showed that the adverse effects of CKD at both individual level and healthcare system level were comparable with those of other major NCDs, and the presence of CKD was associated with poor prognosis of other NCDs. Therefore, CKD should be integrated into the global prevention strategy of NCDs. In consideration of the complexity of the disease, a multilevel interdisciplinary approach will be needed to address the public health burden of CKD.

\section{Author affiliations}

${ }^{1}$ Renal Division, Department of Medicine, Peking University First Hospital, Peking University Institute of Nephrology, Beijing, People's Republic of China

${ }^{2}$ Research Units of Diagnosis and Treatment of Immune-Mediated Kidney Diseases, Chinese Academy of Medical Sciences, Beijing, People's Republic of China

${ }^{3}$ Advanced Institute of Information Technology, Peking University, Hangzhou, People's Republic of China

${ }^{4}$ Clinical Trial Unit, First Affiliated Hospital of Sun Yat-Sen University, Guangzhou,

People's Republic of China

${ }^{5}$ China Standard Medical Information Research Center, Shenzhen, People's Republic of China

${ }^{6}$ Peking-Tsinghua Center for Life Sciences, Beijing, People's Republic of China

${ }^{7}$ National Institute of Health Data Science at Peking University, Beijing, People's Republic of China

${ }^{8}$ Department of Epidemiology, Johns Hopkins University Bloomberg School of Public Health, Baltimore, Maryland, USA

Acknowledgements The authors thank the Bureau of Medical Administration and Medical Service Supervision, National Health Commission of China for the support to this study.

Contributors HW and LZ accept full responsibility for the work as guarantors and/ or the conduct of the study, had access to the data, and controlled the decision to publish. HW and LZ conceived and coordinated the study and acquired the data. $\mathrm{CY}, \mathrm{HW}$ and $\mathrm{LZ}$ designed the study. $\mathrm{CY}$ and $\mathrm{LZ}$ searched the literature. $\mathrm{CY}$ and $\mathrm{JL}$ prepared the figure. CY, HW and LZ wrote the first draft of the manuscript. CY, JL, YS and ZZ contributed to the analysis. CY, JL, YS, ZZ, JW, M-HZ, HW, LZ and JC contributed to the interpretation. CY, JL, YS, ZZ, JW, M-HZ, HW, LZ and JC edited the manuscript. All authors critically reviewed the manuscript and approved the final version.

Funding This study was supported by grants from the National Natural Science Foundation of China (82003529, 91846101, 81771938, 81900665, 82090021), Beijing Nova Programme Interdisciplinary Cooperation Project (Z191100001119008), National Key R\&D Program of the Ministry of Science and Technology of China (2019YFC2005000), Chinese Scientific and Technical Innovation Project 2030 (2018AAA0102100), University of Michigan Health SystemPeking University Health Science Center Joint Institute for Translational and Clinical Research (BMU2018JI012, BMU2019JI005, 71017Y2027), CAMS Innovation Fund for Medical Sciences (2019-I2M-5-046), and PKU-Baidu Fund (2019BD017, 2020BD032).

Competing interests JC was partly supported by grants to the CKD Prognosis Consortium by the National Kidney Foundation and NIH.

Patient consent for publication Not required.

Ethics approval This study involves human participants and was approved by the Ethics Committee of Peking University First Hospital (2015-928). Informed consent was waived by the ethics committee.

Provenance and peer review Not commissioned; externally peer reviewed.

Data availability statement Data may be obtained from a third party and are not publicly available. The data that support the findings of this study are available from the Bureau of Medical Administration and Medical Service Supervision, National Health Commission of China, but restrictions apply to the availability of these data, which were used under licence for the current study and so are not publicly available. Data are however available from the authors upon reasonable request and with permission of the Bureau of Medical Administration and Medical Service Supervision, National Health Commission of China.

Supplemental material This content has been supplied by the author(s). It has not been vetted by BMJ Publishing Group Limited (BMJ) and may not have been peer-reviewed. Any opinions or recommendations discussed are solely those of the author(s) and are not endorsed by BMJ. BMJ disclaims all liability and responsibility arising from any reliance placed on the content. Where the content includes any translated material, BMJ does not warrant the accuracy and reliability of the translations (including but not limited to local regulations, clinical guidelines, terminology, drug names and drug dosages), and is not responsible for any error and/or omissions arising from translation and adaptation or otherwise.

Open access This is an open access article distributed in accordance with the Creative Commons Attribution Non Commercial (CC BY-NC 4.0) license, which permits others to distribute, remix, adapt, build upon this work non-commercially, and license their derivative works on different terms, provided the original work is properly cited, appropriate credit is given, any changes made indicated, and the use is non-commercial. See: http://creativecommons.org/licenses/by-nc/4.0/.

\section{ORCID iDs}

Chao Yang http://orcid.org/0000-0001-9682-8395

Jinwei Wang http://orcid.org/0000-0003-0128-8408

\section{REFERENCES}

1 Beaglehole R, Bonita R, Horton R, et al. Priority actions for the noncommunicable disease crisis. Lancet 2011;377:1438-47.

2 Hunter DJ, Reddy KS. Noncommunicable diseases. N Engl J Med 2013;369:1336-43.

3 Organization WH. Noncommunicable diseases progress monitor 2020, 2020.

4 GBD Chronic Kidney Disease Collaboration. Global, regional, and national burden of chronic kidney disease, 1990-2017: a systematic analysis for the global burden of disease study 2017. Lancet 2020;395:709-33.

5 Eckardt K-U, Coresh J, Devuyst O, et al. Evolving importance of kidney disease: from subspecialty to global health burden. Lancet 2013;382:158-69.

6 Foreman KJ, Marquez N, Dolgert A, et al. Forecasting life expectancy, years of life lost, and all-cause and cause-specific mortality for 250 causes of death: reference and alternative scenarios for 2016-40 for 195 countries and territories. Lancet 2018;392:2052-90.

7 Zhang L, Wang F, Wang L, et al. Prevalence of chronic kidney disease in China: a cross-sectional survey. Lancet 2012;379:815-22. 
8 Yang C, Gao B, Zhao X, et al. Executive summary for China kidney disease network (CK-NET) 2016 annual data report. Kidney Int 2020;98:1419-23.

9 Jha V, Garcia-Garcia G, Iseki K, et al. Chronic kidney disease: global dimension and perspectives. Lancet 2013;382:260-72.

10 Couser WG, Remuzzi G, Mendis S, et al. The contribution of chronic kidney disease to the global burden of major noncommunicable diseases. Kidney Int 2011;80:1258-70.

11 Coresh J, Selvin E, Stevens LA, et al. Prevalence of chronic kidney disease in the United States. JAMA 2007;298:2038-47.

12 Go AS, Chertow GM, Fan D, et al. Chronic kidney disease and the risks of death, cardiovascular events, and hospitalization. $N$ Engl J Med 2004;351:1296-305.

13 Lee M, Saver JL, Chang K-H, et al. Low glomerular filtration rate and risk of stroke: meta-analysis. BMJ 2010;341:c4249.

14 Herzog CA, Asinger RW, Berger AK, et al. Cardiovascular disease in chronic kidney disease. A clinical update from kidney disease: improving global outcomes (KDIGO). Kidney Int 2011;80:572-86.

15 Cai Q, Mukku VK, Ahmad M. Coronary artery disease in patients with chronic kidney disease: a clinical update. Curr Cardiol Rev 2013;9:331-9.

16 Zhang L, Long J, Jiang W, et al. Trends in chronic kidney disease in China. N Engl J Med 2016;375:905-6.

17 Zhang L, Zhao M-H, Zuo L, et al. China kidney disease network (CKNET) 2016 annual data report. Kidney Int Supp/ 2020;10:e97-185.

18 Hirode G, Saab S, Wong RJ. Trends in the burden of chronic liver disease among hospitalized us adults. JAMA Netw Open 2020;3:e201997.

19 Huang Y-M, Xu D, Long J, et al. Spectrum of chronic kidney disease in China: a national study based on hospitalized patients from 2010 to 2015. Nephrology 2019;24:725-36.

$20 \mathrm{Ma} \mathrm{Y-C,} \mathrm{Zuo} \mathrm{L,} \mathrm{Chen} \mathrm{J-H,} \mathrm{et} \mathrm{al.} \mathrm{Modified} \mathrm{glomerular} \mathrm{filtration} \mathrm{rate}$ estimating equation for Chinese patients with chronic kidney disease. J Am Soc Nephrol 2006;17:2937-44.

21 Levine GN, Bates ER, Bittl JA, et al. 2016 ACC/AHA guideline focused update on duration of dual antiplatelet therapy in patients with coronary artery disease: a report of the American College of Cardiology/American heart association Task force on clinical practice guidelines: an update of the 2011 ACCF/AHA/SCAl guideline for percutaneous coronary intervention, 2011 ACCF/AHA guideline for coronary artery bypass graft surgery, 2012 ACC/AHA/ACP/AATS/ PCNA/SCAI/STS guideline for the diagnosis and management of patients with stable ischemic heart disease, 2013 ACCF/AHA guideline for the management of ST-elevation myocardial infarction, 2014 AHA/ACC guideline for the management of patients with non-ST-elevation acute coronary syndromes, and 2014 ACC/ AHA guideline on perioperative cardiovascular evaluation and management of patients undergoing noncardiac surgery. Circulation 2016;134:e123-55

22 Wong CKH, Chen J, Fung SKS, et al. Direct and indirect costs of end-stage renal disease patients in the first and second years after initiation of nocturnal home haemodialysis, hospital haemodialysis and peritoneal dialysis. Nephrol Dial Transplant 2019;34:1565-76.

23 Becker BN, Luo J, Gray KS, et al. Association of chronic condition special needs plan with hospitalization and mortality among patients with end-stage kidney disease. JAMA Netw Open 2020;3:e2023663.

24 Wang V, Vilme H, Maciejewski ML, et al. The economic burden of chronic kidney disease and end-stage renal disease. Semin Nephrol 2016;36:319-30.

25 Saran R, Robinson B, Abbott KC, et al. Us renal data system 2019 annual data report: epidemiology of kidney disease in the United States. Am J Kidney Dis 2020;75:A6-7.

26 Darlington O, Dickerson C, Evans M, et al. Costs and healthcare resource use associated with risk of cardiovascular morbidity in patients with chronic kidney disease: evidence from a systematic literature review. Adv Ther 2021;38:994-1010.

27 Chronic Kidney Disease Prognosis Consortium, Matsushita K, van der Velde $\mathrm{M}$, et al. Association of estimated glomerular filtration rate and albuminuria with all-cause and cardiovascular mortality in general population cohorts: a collaborative meta-analysis. Lancet 2010;375:2073-81.

28 Mahmoodi BK, Matsushita K, Woodward M, et al. Associations of kidney disease measures with mortality and end-stage renal disease in individuals with and without hypertension: a meta-analysis. Lancet 2012;380:1649-61.

29 Fox CS, Matsushita K, Woodward M, et al. Associations of kidney disease measures with mortality and end-stage renal disease in individuals with and without diabetes: a meta-analysis. Lancet 2012;380:1662-73.

30 Stevens PE, Levin A, Kidney Disease: Improving Global Outcomes Chronic Kidney Disease Guideline Development Work Group Members. Evaluation and management of chronic kidney disease: synopsis of the kidney disease: improving global outcomes 2012 clinical practice guideline. Ann Intern Med 2013;158:825-30.

31 Foster MC, Rawlings AM, Marrett E, et al. Cardiovascular risk factor burden, treatment, and control among adults with chronic kidney disease in the United States. Am Heart J 2013;166:150-6.

32 Charytan DM, Wallentin L, Lagerqvist B, et al. Early angiography in patients with chronic kidney disease: a collaborative systematic review. Clin J Am Soc Nephrol 2009;4:1032-43.

$33 \mathrm{Ix} \mathrm{JH}$, Mercado N, Shlipak MG, et al. Association of chronic kidney disease with clinical outcomes after coronary revascularization: the arterial revascularization therapies study (arts). Am Heart $J$ 2005:149:512-9.

34 Kaneko H, Yajima J, Oikawa Y, et al. Role of arterial stiffness and impaired renal function in the progression of new coronary lesions after percutaneous coronary intervention. Cardiovasc Interv Ther 2013;28:56-62.

35 Chen TK, Knicely DH, Grams ME. Chronic kidney disease diagnosis and management: a review. JAMA 2019;322:1294-304. 